\title{
Multi-Criterion Decision Making for Wireless Communication Technologies Adoption in IoT
}

\author{
Abhinav Juneja, BMIET, Sonepat, India \\ iD https://orcid.org/0000-0003-1984-0125 \\ Sapna Juneja, BMIET, Sonepat, India \\ Vikram Bali, JSS Academy of Technical Education, Noida, India \\ iD https://orcid.org/0000-0002-2809-8455 \\ Sudhir Mahajan, Panipat Institute of Engineering and Technology, India
}

\begin{abstract}
There is an unprecedented growth of internet and internet-based technologies in the recent times. We are now switching to $5 \mathrm{G}$ as the most recent wireless communication technology. The internet of things has become a blessing for Industry 4.0 by challenging all the existing technologies in its utility for contributing to the industrial growth. There are a lot of wireless communication technologies for IoT, and it becomes difficult choice to select one suitable for an application. Authors have presented multi-criteria decision-making techniques which are very instrumental in making a confirmed decision on the choice of appropriate technology. This choice is done based on a number of deciding parameter which are used to differentiate between all the available options. The authors have identified 11 wireless communication technologies and seven parameters to evaluate the performance of the WCT's. All the seven parameters are considered in ranking and rank matrix is obtained. This technique can be very helpful for application designers so as to choose the right platform for their applications.
\end{abstract}

\section{KEYWORDS}

Absolute Rank, Hasse Average Rank, Internet of Things, Multi-Criterion Decision Making, Simple Additive Ranking, Wireless Communication Technologies

\section{INTRODUCTION}

In this era of technological advancement, we are facing massive disruption in all domains of our existing Industrial setup. Lampropoulos et al. (2019) explored that there is a lot of quest for the changes next industrial revolution, Industry 4.0 is going to have on our current state of practice. The most significant change that we have already started facing is role of internet and omnipresence of 
human reach due to it. Internet of Things (IoT) has started expanding its wings in all spheres of Human Technological progression. There has been a wide acceptance of IoT driven technology in the recent past, as instanced by Alam (2018), there will be 75.44 billion connected devices by the year 2021 . The internet has expanded its wings and is now collaborating with data science and artificial intelligence to make our machines even smarter and talking to us all the time. It is a new era of thinking about smart machines and smarter technologies. The world is changing the dimensions of communication through use of IoT and smarter technologies. There are various wireless technologies available for communication in IoT and even newer technologies are evolving with changing needs of the market. Each of the technology has inherent advantages and limitations. There are various factors that impact the use of one technology over the other in terms of security, availability, applicability, reliability etc. It becomes a very complex task to take a decision on adoption of one technology over other as all technologies are competing with each other on their specific advantages to support the applications. In this paper the authors have presented a multi criterion decision making approach based on Hasse diagrams presented by Mao (2010) and Voigt et al. (2006), absolute reference discussed in research work of Keller et al. (1991), Hendriks et al. (1992) and average ranking techniques explored by Lewis et al. (1992) to select the best Wireless Communication Technology (WCT) from available options. This approach may be very instrumental for taking into account the impact of each of the contributing factors for choosing the right technology.

\section{LITERATURE REVIEW}

WCT has been the most widely used media for communication in the recent times replacing the old wired communication systems which suffered from the basic limitation to reach the remote areas. Gomez et al. (2010) explored various wireless various home automation protocols and architectures. Hussain et al. (2017) in their work identified the internet of things building blocks. Johari (2015) has discussed the various protocols for IoT communication. Garcia et al. (2018) concluded that popular Wireless options including WiFi, ZigBee, Bluetooth, WiMax, Z wave, NFC have grown as acceptable solutions to the IoT based communication requirements of current Smart City initiatives. Ramadan \& Altamimi (2017) have used IoT wireless networks for a case study of disease production and the efficiency lies on how network forwards and interprets data. Waleed et al. (2016) discuss about the scope and opportunities of wireless $5 \mathrm{G}$ technologies in the Internet of Things and highlight the need of security in the Internet of Things as a major stakeholder to establish trust in adoption of wireless 5G networks. Pau et al. (2018) in their work summed up various available options for the wireless domain to cater to the needs of IoT. Authors summed up that every application domain needs specific requirements relating to the range, throughput, power consumption, and network topology. Besides, further considerations include the cost, ease of integration, and security. In Dhanda et al. (2019), the authors state that the outcome of a reliable communication broadly depends on the availability and reliability of the IoT application. The requirements pertaining to scalability and heterogeneity stage striking challenges to the research community. Mobility maintenance, packet delay and signal load are the primary attributes that inhibit reliable communication and affect the quality of service parameters in IoT. This paper presents the various types of wireless technologies available for the IoT and discusses the open challenges and research issues in IoT. Salman et al. (2017) in their research gave a brief overview of all the protocols available for IoT communication. A similar paper by Krejci et al. (2017) provides a comparative analysis to the developers, designers and the service felicitates with options for various layers of protocols specific for IOT and criteria's of how to choose between them. In Sabaei et al. (2015) the authors have made a comprehensive effort to identify the decision making in an environment where multiple attributes are required to be taken into consideration for decision making. Galli (2019) gave a system engineering approach to decision making using 17 evaluation criteria and gave some newer techniques to make a better decision in multiple parameters affecting the final outcome. Koksalan et al. (2011) presented a detailed analytical introduction to 
Multi Criteria decision (MCDM) making and its advantages. Jha et al. (2012) presents an overview and future directions for component selection in designing fault tolerant modular software systems . Dutta (2017) used a fuzzy approach for decision making in disease diagnosis. Naugle et al. (2019) presented a systems dynamics approach for rational decision making under the influence of various contributing parameters. Jha et al.(2014) gave framework on MCDM for optimal component selection based on cohesion and coupling for component based Software System.Kumar et al. (2016) explored the utility of multi criteria decision making (MCDM) for renewable energy. Shukla et al. (2019) have used a grey scale approach for efficient decision making in for sustainable development in marble industry. Different MCDM methods, their applications, strength and weaknesses have been discussed in the paper. The methods discussed include customized weighted sum method, a weighted product technique, analytical hierarchy process (AHP), elimination with choice translating reality, a Technique for creating preference of order by similarity of ideal solutions. There are many more such methods which define a solution for finding the best available solution to a decision making problem. Bali and Madan (2015) presented a study called TOPVEC Framework of critical success factors in COTS based software development Patil et al. (2004) presented the concept of partial ordered sets and linear extensions to make a decision of choice between multiple indicators. Voigt et al. (2006) evaluated multiple decision making indicators using Hasse Diagram Techniques (HDT) which originates from discrete mathematics. In Carlsen (2009), Tsonkova et al. (2015) and Carlsen (2015), the authors have presented how Hasse diagrams, partial order ranking techniques and the linear extensions can be given an equal opportunity to all the attributes making a decision be represented in an unbiased manner and allowing the decision in such an environment be made with consideration of all the present attributes. From the review of existing literature it may be enumerated that there is a wide range of wireless communication technologies available in today's scenario, these technologies may be applicable even to a single application in IoT, Carlson (2019),(2015) emphasized that we need to device a mechanism to choose between these existing technologies, linear extensions and total order ranking is very efficient technique to identify and rank these technologies for a particular application based on its requirements.

\section{DIFFERENT IOT WIRELESS COMMUNICATION TECHNIQUES (WCT)}

In the current era a number of wireless technologies at data link layer exist for the application designers and each of these technologies have their own specific attributes that make them suitable for use and attract the application designers. The major advantage of WCT includes no use of guiding cables, dependability, security, safety and many more. The various technologies adopted for Wireless Communication in IoT devices have been discussed by Salman et al. (2017), Alsharif et al. (2019), Kogias et al. (2019), Wang et al. (2018), Huynh et al. (2017) and Ghamari et al. (2015) in their respective research work. Authors have considered following 11 WCT's for selection of most suitable IoT communication based applications.

\section{WiFi}

Wireless Fidelity also called as Wifi is one of the widely used wireless technologies for connecting the electronic devices in wireless area networks (WAN). The Wifi technologies is based on IEEE 802.11standard that has an operational band in the range of $2.4 \mathrm{Ghz}$ to $5 \mathrm{Ghz}$. This is an unlicensed band available worldwide for such usage, Anani et al. (2019). Due to the availability of high bandwidth, Wifi is capable of providing high data transfer rates in IoT Devices but it suffers from a limitation of higher power consumption compared to its competitor technologies.

\section{Bluetooth}

This is based on the IEEE 802.15.1 standard. It is employed for short range communication between static as well as mobile devices in a personal area network (PAN). It employs a low cost transceiver 
microchip and utilizes the band of $2.4 \mathrm{Ghz}$. The Bluetooth technology also shares the common free license zone with other technologies, Dhanda (2019). Bluetooth 5 delivers four variants of data rates in order to cater to a variety of transmission ranges: $2 \mathrm{Mbps}, 1 \mathrm{Mbps}, 500 \mathrm{kbps}$, and $125 \mathrm{kbps}$ in Bluetooth version 5.0.

\section{Bluetooth Low Energy (BLE)}

It is also called Bluetooth smart and was introduced in Bluetooth version 4.0. This technology is targeted towards the applications relying on lower power consumption, BLE provides a lower data throughput of 1Mbps utilizing GFSK modulation scheme. Occasionally BLE's max data throughput of $1 \mathrm{Mbps}$ may not be suit the products which need a continuous streaming of data like wireless headphones, while other IoT applications only need to send small bits of data periodically.

\section{Z Wave}

This wireless technology generally finds its application in home automation. Z-wave technology enables devices "mesh" together with each other by sending signals over low-energy radio waves on a dedicated frequency for transmission. Every Z-wave device possesses a small built-in signal repeater that sends and receives network information. $Z$ wave offers better signal transfer and reception compared to its competing technologies due to repeaters present in each device in the network and making the network even stronger.

\section{Near Field Communication (NFC)}

This is a very short range (less than $10 \mathrm{~cm}$ ) communication protocol. Generally used in applications like contactless payments including credit cards, smart cards, e ticketing etc, Sethi (2017). This distance limit ensures much safer communication among devices while it needs more time as the devices are needed to be moved physically in close proximity to make a communication or transaction possible.

\section{Zigbee}

This is a low power, low data rate transmission technique usually finds in applications where we need to have interoperability between devices which are from different manufacturers. Zigbee is an open source protocol and all the manufacturers may use it in their applications, Sethi (2017). Zigbee is based on IEEE 802.15.4 standard. It uses three unlicensed bands for communication.

\section{ANT}

It is an ultra- low power transmission protocol. The device using this technology can run on a coin cell for years of operation. This is a self adaptive technology which is capable of making quick meshes. It required a low cost design and operates with a single chip.

\section{DASH7}

This is recent wireless communication protocol that is primarily used in active RFID devices, generally functions in globally accessible industrial scientific medical band. Ayoub et al. (2018) stated that it has been specifically targeted for scalable and long-range outdoor implementation having much better data rates than the popular ZigBee. This is an affordable protocol that enforces encryption and implements IPv6 addressing. It targets bursty, light weighted, asynchronous and on the move traffic and hence typically suits IoT applications.

\section{Home Plug}

Home plug greenphy was developed by home plug powerline alliance and generally employed in home automation. This WCT has been primarily focussed on reducing the power consumption and affordability of HomePlug-AV additionally providing its reliability, availability, coverage and 
interoperability. It supports a power-save operational profile that permits the nodes to sleep by synchronizing their respective sleep time and waking them up whenever needed.

\section{EnOcean}

EnOcean is a wireless communication technology with prime focus on energy conservation, it is very often implemented in automation based applications, yet has potential to be utilized for other IoT applications. The fundamental approach is to harvest motion or any other type of environmental energy efficiently and then convert it to any form of usable energy by employing specific transducers. EnOcean protocol provides small packet size and is employed purposes including cooling, ventilation, and heat based IoT applications.

\section{LoRaWAN}

LoRaWAN is a recently introduced long-range protocol for application in wide-area networks. This particular WCT has been purposely designed to support IoT applications enabling power economy, affordability, dynamics, reliability, and duplex communication requirements. It is designed for wireless networks that can be scaled to a capacity of millions of devices, Poursafar (2017). It enables redundant operation, power optimization, affordable, mobile, and energy procuring systems to shape the upcoming requirements of IoT along with supporting the mobility and easy to use features.

Table 1 shows the technical specifications of the various wireless communication technologies used in the modern day Internet of Things (IoT) applications.

\section{WCT SELECTION CRITERIA}

There are a number of criteria's which need to be taken into account for selecting a particular wireless communication technology for a specific application. The various technologies have been compared on their key performance attributes by Lee et al. (2007). Kondratenko et al. (2018) has proposed several parameters to select a particular technology over other for WCT's. Elfouly et al. (2017) have insisted that for an efficient WSN energy consumption is one of the most important parameter along with other parameters. Several technologies may appear to suit a particular application but we need to analyze each of these technologies on some specific attributes and then come on a decision of selecting a particular WCT. The various specific attributes to be taken into consideration are:

Table 1. Various popular IOT Wireless Technologies in use

\begin{tabular}{|l|l|l|l|}
\hline \multicolumn{1}{|c|}{ WCT Technology } & \multicolumn{1}{c|}{ Range } & \multicolumn{1}{c|}{ Data Rate } & \multicolumn{1}{c|}{ Frequency } \\
\hline WiFi & $100 \mathrm{Mts}$ & $54 \mathrm{Mbps}$ & $2.4 \mathrm{Ghz}$ and $5 \mathrm{Ghz}$ \\
\hline Bluetooth & $10 \mathrm{Mts}$ & $24 \mathrm{Mbps}$ & $2.4 \mathrm{Ghz}$ \\
\hline Bluetooth Low Energy & $8-10 \mathrm{Mts}$ & $1 \mathrm{Mbps}$ & $2.4 \mathrm{Ghz}$ \\
\hline Z Wave & $30 \mathrm{Mts}$ & $100 \mathrm{Kbps}$ & $900 \mathrm{Mhz}$ \\
\hline Near Field Communication & Upto $10 \mathrm{~cm}$ & $424 \mathrm{Kbps}$ & $13.56 \mathrm{Mhz}$ \\
\hline Zigbee & $100-1500 \mathrm{Mts}$ & $250 \mathrm{Kbps}$ & $868 \mathrm{Mhz}, 915 \mathrm{Mhz}, 2.4 \mathrm{Ghz}$ \\
\hline ANT & $30 \mathrm{Mts}$ & $60 \mathrm{Kbps}$ & $2.4 \mathrm{Ghz}$ \\
\hline DASH7 & $1-2 \mathrm{Kms}$ & $200 \mathrm{Kbps}$ & $868 \mathrm{Mhz}, 915 \mathrm{Mhz}$ \\
\hline Home Plug & $200 \mathrm{Mts}$ & $200 \mathrm{Mbps}$ & $4.5-21 \mathrm{Mhz}$ \\
\hline EnOcean & $300 \mathrm{Mts}$ & $125 \mathrm{Kbps}$ & $315 \mathrm{MHz}, 868 \mathrm{Mhz}, 900 \mathrm{Mhz}$ \\
\hline LoRaWAN & $<30 \mathrm{Kms}$ & $.3-50 \mathrm{Kbps}$ & $868 \mathrm{Mhz}, 900 \mathrm{Mhz}$ \\
\hline
\end{tabular}


1. Reliability: Reliable data transfer has a direct impact on the user transfer and the battery life of the device. If there is a situation of undeliverable packet to the destination due to reasons including interference from adjacent channels, deliberate frequency jamming etc. then the transmitter keeps on trying to deliver the packet till it it delivered on the expense of battery.

2. Security: WEP and WPA2 are the standards being in force to ensure the security of data on wireless networks. Some of the hardware equipments find it difficult to adopt WPA2 without firmware updation.

3. Range: Higher transmission power enables better range due to high signal to power ratio of transmission. Regulatory bodies control the maximum power output in the license free $2.4 \mathrm{GHz}$ ISM band.D

4. Data Rate: IoT sensor devices generally transfer periodic data to the central controller. This minimizes the power consumption. Bandwidth requirement are typically modest here.

5. Signal Latency: Latency related to the time lapsed between transmission of signal and the reception of signal. Though this lies in the range of milliseconds but becomes very crucial in some of the IoT applications.

6. Power Transfer: Various technologies differ from each other in terms of distance to which power can be propagated by them.

7. Topology: In the current scenario the existing WCTs broadly support four different types of topologies to serve different applications which include peer to peer, broadcast, star and scanning.

\section{SELECTION OF THE MOST SUITABLE IOT WCT}

We have a number of WCTs available and need to give due weightage to a number of evaluation parameters while analyzing the suitable WCT for any application. In our work we have used a noble multi criteria decision making technique for finding out the most suitable WCT. This method demonstrated Juneja et al. (2019) takes into account the significance of all the contributing attributes in the selection of a WCT. We have used Simple Additive ranking, Hasse Diagrams presented by Mao (2010) and Voigt et al. (2006), Carlsen (2009) and Carlsen (2015) and Absolute reference mentioned by Keller et al. (1991) and Hendriks et al. (1992) for ranking the WCTs for suitability of a particular application.

\section{Simple Additive Ranking (SAR)}

SAR technique provides ranking to the objects taking into account each criterion separately and subsequently ranks the succeeding aggregation of the weighted ranks using the arithmetic mean. Ranking $\mathrm{r}_{\mathrm{ij}}$ of $i$-th element corresponding to the $j$-th parameter is calculated, the corresponding index value is generated by Equation 1:

$$
s_{i}=\frac{\sum_{j=1}^{P} w_{j} \cdot r_{i j}}{n}
$$

Then this value is normalized using Equation 2:

$$
S_{i}^{\prime}=\frac{S_{i}-1 / n}{1-1 / n}
$$




\section{Hasse Diagram}

Hasse diagrams visualize the order of relation between the partial order sets (poset). Two objects a and $b$ of a poset are ordered if all attributes of a are less than or greater than that of $b$. Hasse diagrams consists of a directed graph which consists of a set $\mathrm{E}$ of objects drawn as circles, circles at the top of the graph are the best objects to suit a ranking criteria and the ones at the bottom are least suited. These objects have no predecessors and are called as maximal points. A line between two objects in the Hasse diagram represents that two objects under consideration are related.

\section{Partial Order Sets (POSET)}

The partial order creates a ranking relation between several elements simultaneously considering multiple parameters of the elements. Two elements are said to be comparable if all attributes of one element (a1) are less than or equal to or more than the corresponding attributes of the other (a2). If the element a1 has 3 attributes $(4,6,8)$ and element a2 has three similar attributes with values $(1,2$, $3)$. Figure 1 shows attributes A1, A2 and A3 of two elements a1 and a2. It is very clear from Figure 1 that $\mathrm{a} 1>\mathrm{a} 2$ as all attributes of a1 are greater than that of $\mathrm{a} 2$.

Figure 2 shows that element a1 is bigger than element a2 with the help of a Hasse Diagram. But if for the elements a3 $(3,5,4)$ and a4 $(2,4,7)$, we try to create an order then these two elements become incomparable due to their incomparable attribute combinations. So in order to sequence these elements a1, a2, a3 and a4, we create a partial order also called a poset. The most crucial factor in sequencing a poset is the number of comparisons which may be actuated. It is expected to gather as much comparisons as feasible as each present incomparability of the poset represents one corresponding indetermination. The comparison that may be realized is dependent on number of attributes which are needed to be taken into consideration for the purpose of creating order. On addition of new attributes to the poset, there will be a decrease in the comparisons this leads to increase in the degree of indetermination. In poset having a considerably huge number of elements

Figure 1. Attributes $A 1, A 2$ and $A 3$

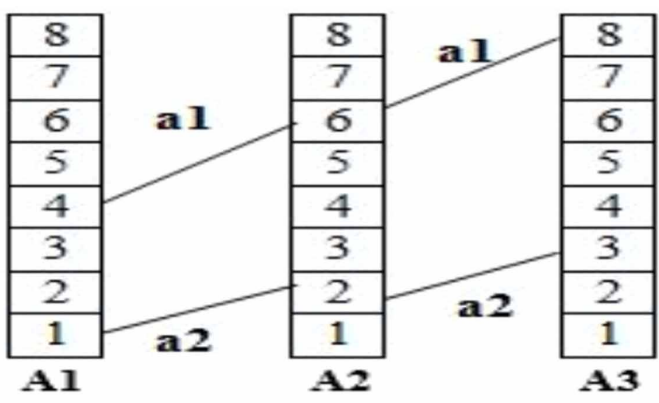

Parallel Coordinates

Figure 2. Hasse diagram for element a1and a2

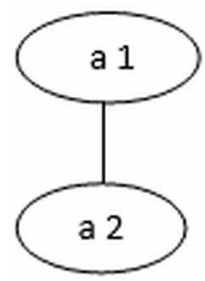


and uncorrelated attributes, the quantum of comparisons shrink to half whenever an attribute is added as stated by Lebanon et al. (2002), Peter et al. (2003) and Restrepo et al. (2006), they have used the same concept in ranking posets in their respective research.

\section{Average Rank and Total Order}

Average rank determination can be realized through the use of the local partial order. Average Ranking is done through an empirical relation relying on linear extensions of partial order. Linear extension is typically prediction of partial order spawning a total order that conforms for all relations present in partial order. Therefore, from a partial order ranking it is feasible to estimate linear extensions, and correspondingly evaluate an average ranking of each element based on its ranking frequencies for all linear extensions. Consequently, the averaged rank of the element $\mathrm{x}$ can be valued by using the following formula presented by Bruggemann et al. (2004) and Bruggemann et al. (2011):

$$
\mathrm{Rk}_{(\mathrm{av})}=(\mathrm{S}+1) *(\mathrm{~N}+1) /(\mathrm{N}+1-\mathrm{U})
$$

where $\mathrm{S}$ is the aggregate number of descendants of the element $\mathrm{x}, \mathrm{N}$ is the number of elements (of the residual quotient set), and $\mathrm{U}$ is the range of elements incomparable with $\mathrm{x}$.

\section{Absolute Reference}

The absolute reference presented in their work by Keller et al. (1991) and Hendriks et al. (1992) measures the displacement between each element into consideration and corresponding element of reference, which represents overall optimum of all the criteria taken into consideration. In absolute reference, we need the characterisation of the values and conditions of optimum, i.e. for each criterion it is necessary to unambiguously establish not only whether the best condition is fulfilled with a minimum value or a maximum value of the criterion, moreover the specific optimum values. Each criterion first prerequisites to be normalized and weighed to account for its significance. After opting the distance measure, the method calculates the overall $\mathrm{N}$ distances among elements under consideration and the reference element. Here Euclidean distance is used, distance of $\mathrm{i}$-th element from the reference element $\left(i^{*}\right)$ is defined with Equation 4:

$d_{i i}^{*}=\sqrt{\sum_{r=1}^{R}\left(y_{i f}-y_{i^{*}}\right)^{2} \cdot W_{r}}$

Corresponding to every element a representation of its similarity compared to the set reference element is worked out with the help of the Euclidean distance incorporating the following expression:

$\mathrm{S}_{\mathrm{i}}=1-\mathrm{d}_{\mathrm{ii}} *$ where $0 \leq \mathrm{S}_{\mathrm{i}} \leq 1$

This degree of similarity is used for ranking of the elements. It ranges from 0 (if there is no similarity between the considered element and the reference element) and 1 (if there occurs thorough resemblance amongst the considered element and the reference element).

\section{CASE STUDY OF MULTI CRITERIA SELECTION OF WCT}

The wireless communication technologies identified in the Section 3 above are among the most commonly employed media for communication in the IoT based devices. We have taken the criteria mentioned in Section 4 above as the key attributes for any of the wireless communication technology 
for IoT to create a rank matrix. The process adopted by us in our research work has been represented by Figure 3.

We have used partial order, average ranking and linear extensions for ranking the WCT. Here we have considered the identified attributes to make a decision. The attributes are represented by literal P as Reliability(P1), Security(P2), Range(P3), Data Rate(P4), Signal Latency(P5), Power Tranfer(P6), Topology(P7) have been evaluated as a case study for all the prevalent IoT WCT's which are represented by the literal Q as Wifi(Q1), Bluetooth(Q2), Bluetooth Low Energy(Q3),Z wave(Q4), Near Field Communication(Q5), Zigbee(Q6), ANT(Q7), DASH7(Q8), Home Plug(Q9), EnOcean(Q10), LoRaWAN(Q11). We have rated these 7 attributes on a scale of 0 to 9 for an IoT application for all the available WCT's as shown in Table 2. As similar type of approach was followed by considered by Kondratenko et al. (2018) in their work on selecting the ranking parameters.

\section{RESULTS}

The above-mentioned ranking criteria's namely Simple Average Ranking (SAR), Hasse Average Ranking (HAR) and Absolute Reference (AR) have been applied on all the available techniques in Table 2 based on the seven evaluation parameters. The ranking of these models for the three ranking methods is given in Table 3. The models have been ranked based on absolute ranking in this table. It is evident from the ranking table that all the three methods are giving different ranking to different WCTs due to their inherent evaluation technique. Here Simple additive ranking provides a basic ranking focused on weighing each criterion, Hasse average ranking uses posets and average ranking to find the ranking and is able to identify the three levels of ranks that may be assigned to different WCT's.

Figure 3. Process of Identifying the most suitable WCT for loT Application

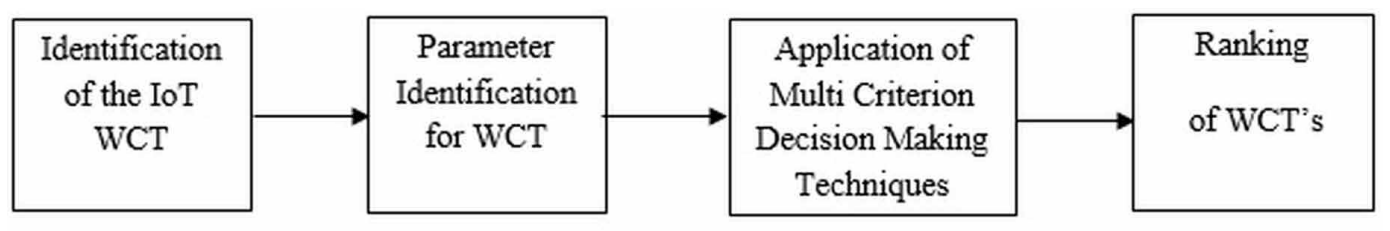

Table 2. WCT's and attribute weights

\begin{tabular}{|c|c|c|c|c|c|c|c|}
\hline WCT/Att. & P1 & P2 & P3 & P4 & P5 & P6 & P7 \\
\hline Q1 & 7 & 9 & 4 & 3 & 5 & 6 & 5 \\
\hline Q2 & 4 & 4 & 7 & 9 & 4 & 8 & 6 \\
\hline Q3 & 5 & 4 & 2 & 4 & 8 & 8 & 4 \\
\hline Q4 & 8 & 9 & 7 & 8 & 6 & 7 & 5 \\
\hline Q5 & 3 & 4 & 8 & 7 & 9 & 5 & 6 \\
\hline Q6 & 1 & 5 & 6 & 6 & 6 & 4 & 3 \\
\hline Q7 & 7 & 3 & 8 & 7 & 7 & 8 & 4 \\
\hline Q8 & 9 & 2 & 9 & 3 & 2 & 5 & 8 \\
\hline Q9 & 5 & 6 & 8 & 3 & 4 & 4 & 1 \\
\hline Q10 & 7 & 8 & 4 & 8 & 2 & 5 & 6 \\
\hline Q11 & 4 & 4 & 8 & 4 & 6 & 3 & 7 \\
\hline
\end{tabular}


Table 3. Ranking of WCT's with multiple ranking techniques sorted by absolute reference

\begin{tabular}{|l|l|l|l|l|}
\hline \multicolumn{1}{|c|}{ Rank } & \multicolumn{1}{c|}{ WCT } & \multicolumn{1}{c|}{ SAR } & \multicolumn{1}{c|}{ HAR } & \multicolumn{1}{c|}{ Absolute Reference } \\
\hline 1 & Q4 & 0.700 & 0.800 & 1.000 \\
\hline 2 & Q10 & 0.528 & 0.500 & 0.678 \\
\hline 3 & Q7 & 0.585 & 0.500 & 0.646 \\
\hline 4 & Q2 & 0.542 & 0.500 & 0.635 \\
\hline 5 & Q1 & 0.485 & 0.300 & 0.631 \\
\hline 6 & Q5 & 0.557 & 0.500 & 0.567 \\
\hline 7 & Q6 & 0.314 & 0.300 & 0.512 \\
\hline 8 & Q3 & 0.485 & 0.500 & 0.500 \\
\hline 9 & Q9 & 0.328 & 0.500 & 0.492 \\
\hline 10 & Q11 & 0.471 & 0.500 & 0.473 \\
\hline 11 & Q8 & 0.499 & 0.500 & 0.407 \\
\hline
\end{tabular}

While the absolute reference calculates the final ranking based on the euclidean distance from the mean value for different decision parameters. The final ranking of various wireless communication technologies based on Absolute rankings represented by Figure 4. The WCT Q4 i.e. Z wave ranks the best WCT's as per the weightage given to its various attributes.

\section{CONCLUSION}

We have evaluated the available wireless techniques for random application environments and used 7 parameters to decide on the choice of a particular WCT among options for implementation of a particular Internet of Things (IoT) application. This approach may be applied to make an informed decision on the selection of an appropriate technology based on various attributes which are inherent requirement for the implementation of any application. Various attributes which are required for any wireless communication technology to be implemented have some of the matching parameters as

Figure 4. Absolute ranking of various technologies based on 7 attributes

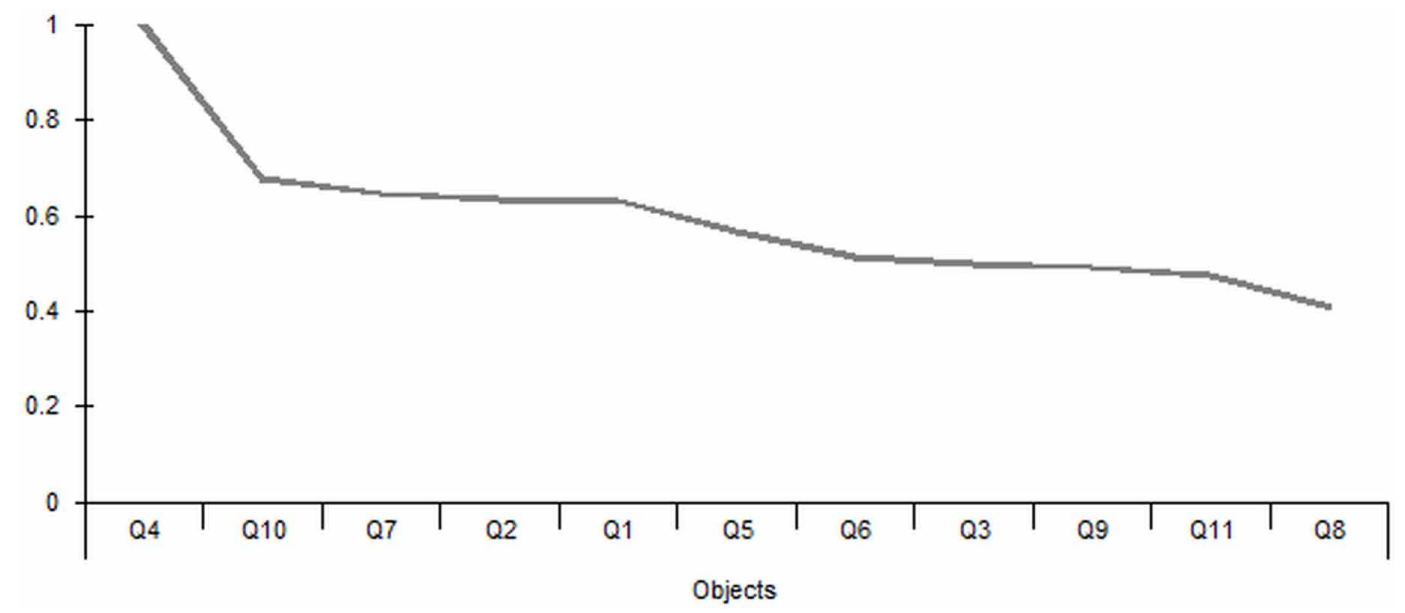


shown in Table 1, in such situation the methodology proposed in this research would be helpful for finding out the actual ranking of the various technologies in terms of the user need set for a particular IoT application. Three multi criteria decision making techniques have been explored here. All the techniques consider the participation of some quantum of the deciding parameters in their ranking of the Wireless Communication Technologies. The Hasse Average ranking technique used here typically is a discrete mathematics approach to rank using graphical structures and give equal weightage to each participating object in the comparisons and draw a relation of hierarchy among various objects under comparison having multiple attributes to compare at the same time. These techniques can be very instrumental in making a resolution on evolution of WCT's for the selection of the applications of Internet of Things. As IoT is an emerging field and there is a lot of effort being made to come out with a standard set of protocol to leverage the technology, in such scenario this methodology may provide more trust to the application designers while making a choice on the WCT. The methods explored in this research work may be used in drawing inferences for making other such crucial decisions where multiple criteria decision making is required and it is very critical to ignore any of the attribute parameters.

Through this work the authors have proposed a methodology which can be used for WCT adoption in IoT still there may be some limitations which can influence these decisions. The priorities assigned by experts to different parameters for different WCT's may be biased due to various technological, financial and personal factors. This may finally lead to selection of inappropriate WCT for the wireless applications. So we need a rational allocation of priorities to various attributes for this. There is a need for standardization of rating for various comparison parameters. One more improvisation that can be added as a future dimensional extension in the current proposed methodology is ranking by weights. We can assign weights to various criteria and allocate them priority while ranking which will make the selection of WCT even more rational. Higher weights may be assigned to those parameters which have major role in the performance measurement and feasibility of the IoT application and lower to those whose role is limited compared to the others. 


\section{REFERENCES}

Alam, T. (2018). Reliable communication framework and its use in Internet of Things (IoT). International Journal of Scientific Research in Computer Science. Engineering and Information Technology, 5, 450-456.

Alsharif, M. H., Kim, S., \& Kuruoğlu, N. (2019). Energy Harvesting Techniques for Wireless Sensor Networks/ Radio-Frequency Identification: A Review. Symmetry, 11(7), 865. doi:10.3390/sym11070865

Anani, W., Ouda, A., \& Hamou, A. (2019). A Survey of Wireless Communications for IoT Echo-Systems. IEEE Canadian Conference of Electrical and Computer Engineering (CCECE), Edmonton, AB, Canada, 1-6. doi:10.1109/CCECE.2019.8861764

Ayoub, W., Samhat, A., Nouvel, F., Mroue, M., \& Prévotet, J. (2018).Internet of Mobile Things: Overview of LoRaWAN, DASH7, and NB-IoT in LPWANs standards and Supported Mobility. 25th International Conference on Telecommunications (ICT), 21(2), 1561-1581.

Bali, V., \& Madan, S. (2015). A Study of Critical Success Factors in COTS based Software Development: TOPVEC Framework. Wulfenia Journal, 22(8), 174-186.

Bruggemann, R., Sorensen, P. B., Lerche, D., \& Carlsen, L. (2004). Estimation of averaged ranks by a local partial order model. Journal of Chemical Information and Computer Sciences, 44(2), 618-625. doi:10.1021/ ci034214m PMID: 15032543

Bruggemann, Rainer, Patil, \& Ganpati. (2011). Ranking and prioritization for multi-indicator systems. Ranking and Prioritization for Multi-indicator Systems.

Bruggemann, R., \& Carlsen, L. (2011). An Improved Estimation of Averaged Ranks of Partial Orders. Match Commun. Math. Comput. Chem., 65, 383-414.

Carlsen, L. (2009). The Interplay between QSAR/QSPR Studies and Partial Order Ranking and Formal Concept Analyses. International Journal of Molecular Sciences, 10(4), 1628-1657. doi:10.3390/ijms10041628 PMID:19468330

Carlsen, L. (2015). Partial ordering as decision support to evaluate remediation technologies. AIMS Environmental Science, 2(1), 110-121. doi:10.3934/environsci.2015.1.110

Dhanda, S. S., Singh, B., \& Jindal, P. (2019). Wireless technologies in IOT: Research challenges, Engineering Vibration. Communication and Information Processing, Lecture Notes in Electrical Engineering, 229-239.

Dutta, P. (2017). Decision Making in Medical Diagnosis via Distance Measures on Interval Valued Fuzzy Sets. International Journal of System Dynamics Applications, 6(4), 63-83. doi:10.4018/IJSDA.2017100104

Elfouly, F. H., Ramadan, R. A., Mahmoud, M. I., \& Dessouky, M. I. (2017). Efficient Data Reporting in a Multi-Object Tracking Using WSNs. International Journal of System Dynamics Applications, 6(1), 38-57. doi:10.4018/IJSDA.2017010103

Galli, B. J. (2019). Thoughts of Using Economic Decision-Making to Systems Engineering and Systems Thinking: An Exploratory Study. International Journal of System Dynamics Applications, 8(3), 1-14. doi:10.4018/ IJSDA.2019070101

Garcia, L., Jimenez, J., Taha, M., \& Lloret, J. (2018). Wireless technologies for IOT in smart cities. Network Protocols and Algorithms, 10(1), 23-64. doi:10.5296/npa.v10i1.12798

Ghamari, M., Arora, H., Sherratt, R., \& Harwin, W. (2015). Comparison of low-power wireless communication technologies for wearable health monitoring applications. International Conference on Computer, Communications, and Control Technology (I4CT), 92-106. doi:10.1109/I4CT.2015.7219525

Gomez, C., \& Paradells, J. (2010). Wireless home automation networks: A survey of architectures and Technologies. Communications Magazine, 48(6), 92-101. doi:10.1109/MCOM.2010.5473869

Hendriks, M. M. W. B., De Boer, J. H., Smilde, A. K., \& Doornbos, D. A. (1992). Multicriteria decision making. Chemometrics and Intelligent Laboratory Systems, 16(3), 175-191. doi:10.1016/0169-7439(92)80036-4

Hussain, F. (2017). Internet of Things: Building Blocks and Business Models. Briefs in Electrical and Computer Engineering, 1. 
Huynh, N., Robu, V., Flynn, D., Rowland, S., \& Coapes, G. (2017). Design and demonstration of a wireless sensor network platform for substation asset management. CIRED - Open Access Proceedings Journal, 2017(1), 105-108.

Jha, P.C., \& Bali, V. (2012). Component Selection in Designing Fault Tolerant Modular Software systems: An Overview and Future Directions. International Journal of Science, Technology and Management, 3(1), 1-8.

Jha, P. C., Bali, V., Narula, S., \& Kalra, M. (2014). Optimal Component Selection Based on Cohesion and Coupling for Component based Software System. Proceedings of the Second International Conference on Soft Computing for Problem Solving (SocProS 2012). doi:10.1007/978-81-322-1602-5_150

Johari, F. (2015). The security of communication protocols used for Internet of Things. Lund University.

Juneja, A., Bajaj, S., \& Anand, R. (2019). Improvising Green Computing Using Multi Criteria Decision Making. Journal of Advanced Research in Dynamical and Control Systems, 12(3), 1161-1165.

Keller, H. R., Mossart, D. L., \& Brans, J. P. (1991). Multicriteria decision making. Chemometrics and Intelligent Laboratory Systems, 16(2), 175-192. doi:10.1016/0169-7439(91)80064-W

Kogias, D.G.,Michailidis, E.T., Tuna, G., \& Gungor, V.V. (2018). Realizing the wireless technology in internet of things (IOT). Emerging Wireless Communication and Network Technologies, 173-192.

Koksalan, M., Wallenius,J., \& Zionts, S.(2013). An Early History of Multiple Criteria Decision Making, Journal of Multi-Criteria Decision Analysis, 20.

Kondratenko, I. S. Y., \& Kondratenko, G. (2018). Multi-criteria selection of the wireless communication technology for specialized iot network. ICTERI Workshop, 245.

Krejčí, R., Hujňák, O., \& Švepeš, M. (2017). Security survey of the iot wireless protocols. Proceedings of the 25th Telecommunication Forum (TELFOR), 1-4 doi:10.1109/TELFOR.2017.8249286

Kumar, A., Sah, B., Singh, A., Deng, Y., He, X., Kumar, P., \& Bansal, R. (2016). A review of multi criteria decision making $(\mathrm{mcdm})$ towards sustainable renewable energy development. Renewable \& Sustainable Energy Reviews, 69, 596-609. doi:10.1016/j.rser.2016.11.191

Lampropoulos, G., Siakas, K., \& Anastasiadis, T. (2019). Internet of things in the context of Industry 4.0: An overview. International Journal of Entrepreneurial Knowledge, 7(1), 4-19. doi:10.37335/ijek.v7i1.84

Lebanon, G., \& Lafferty, J. (2002). Conditional models on the ranking poset. NIPS'02: Proceedings of the 15th International Conference on Neural Information Processing Systems, 431-438.

Lee, J., Su, Y., \& Shen, C. (2007). A Comparative Study of Wireless Protocols: Bluetooth, UWB, ZigBee, and Wi-Fi. IECON 2007 - 33rd Annual Conference of the IEEE Industrial Electronics Society, 46-51.

Lewi, P. J., Van Hoof, J., \& Boey, P. (1992). Multicriteria decision making using Pareto Optimality and Promethee Prefrence Ranking. Chemometrics and Intelligent Laboratory Systems, 16(2), 139-144. doi:10.1016/01697439(92)80052-6

Mao, S. (2010). Algorithm for Weighted Hasse Diagram and its Application. International Conference on Computational Intelligence and Software Engineering, 1-4. doi:10.1109/CISE.2010.5677073

Naugle, A. B., Backus, G. A., Tidwell, V. C., Kistin-Keller, E., \& Villa, D. L. (2019). A Regional Model of Climate Change and Human Migration. International Journal of System Dynamics Applications, 8(1), 1-22. doi:10.4018/IJSDA.2019010101

Patil, G., \& Taillie, C. (2004). Multiple indicators, partially ordered sets,and linear extensions: Multicriterion ranking and prioritization. Environmental and Ecological Statistics, 11(2), 199-228. doi:10.1023/ B:EEST.0000027209.93218.d9

Pau, G., Chaudet, C., Zhao, D., \& Collotta, M. (2018). Next Generation Wireless Technologies for Internet of Things. Sensors (Basel), 18(1), 221. doi:10.3390/s18010221 PMID:29342892

Peter, B. (2003). Analysis of monitoring data of pesticide residues in surface waters using partial order ranking theory. Environmental Toxicology and Chemistry, 22(3), 661-670. doi:10.1002/etc.5620220327 PMID:12627656 
Poursafar, N., Alahi, M. E. E., \& Mukhopadhyay, S. (2017). Long-range wireless technologies for iot applications: A review. Eleventh International Conference on Sensing Technology (ICST), 1-6. doi:10.1109/ ICSensT.2017.8304507

Ramadan, R., \& Altamimi, A. B. (2017). Hierarchal Fuzzy Logic Controller and Internet of Things (IoT) Information: Disease Spreading as a Test Case. International Journal of System Dynamics Applications, 6(3), 59-86. doi:10.4018/IJSDA.2017070104

Restrepo, G., \& Bruggemann, R. (2006). Ranking regions using cluster analysis, Hasse diagram technique and topology. 3rd International Conference on environmental modeling and software.

Sabaei, D., Erkoyuncu, J., \& Roy, R. (2015). A review of multi-criteria decision making methods for enhanced maintenance delivery. Procedia, CIRP, 37, 30-35. doi:10.1016/j.procir.2015.08.086

Salman,T., \& Jain R. (2017). A survey of protocols and standards for internet of things. Advanced Computing and Communications, $1(1)$.

Sethi, P., \& Sarangi, R. S. (2017). Internet of things: Architectures, Protocols, and Applications. Journal of Electrical and Computer Engineering, 2017, 1-25. doi:10.1155/2017/9324035

Shukla, O. J., Jangid, V., Soni, G., \& Kumar, R. (2019). Grey Based Decision Making for Evaluating Sustainable Performance of Indian Marble Industries. International Journal of System Dynamics Applications, 8(2), 1-18. doi:10.4018/IJSDA.2019040101

Tsonkova, P., Böhm, C., Quinkenstein, A., \& Freese, D. (2015). Application of partial order ranking to identify enhancement potentials for the provision of selected ecosystem services by different land use strategies. Agricultural Systems, 135, 112-121. doi:10.1016/j.agsy.2015.01.002

Voigt, K. S. P., Bruggemann, R., \& Pudenz, S. (2006). A multi-criteria evaluation of environmental databases using the Hasse diagram Technique (pro-rank) Software, Computer Science. Environmental Modelling \& Software, 21(11), 1587-1597. doi:10.1016/j.envsoft.2006.05.005

Waleed, E., Muhammad, A. I., Minho, J., \& Anpalagan, A. (2016). Internet of Things (IoT) in 5G Wireless Communications. IEEE Access: Practical Innovations, Open Solutions, 4, 10310-10314. doi:10.1109/ ACCESS.2016.2646120

Wang, C., Chen, S., Yang, Y., Hu, F., Liu, F., \& Wu, J. (2018). Literature review on wireless sensing-Wi-Fi signal-based recognition of human activities. Tsinghua Science and Technology, 23(2), 203-222. doi:10.26599/ TST.2018.9010080 


\begin{abstract}
Abhinav Juneja $(P h D)$ is currently working as Associate Director \& Professor at BMIET, Sonepat. He has more than 18 years of teaching experience for teaching post graduate and under graduate engineering students. He completed his Doctorate in Computer Science and Engineering from M.D. University, Rohtak in 2018 and has done masters in Information Technology from GGSIPU, Delhi. He has research interests in the field on Software Reliability, IoT, Machine Learning and soft computing. He has published several papers in reputed national and international journals. He has been mentor of several post graduate and under graduate research oriented projects.
\end{abstract}

Sapna Juneja $(P h D)$ is Professor in Department of Computer Science and Engineering at BMIET, Sonepat. She has more than 15 years of teaching experience. She completed her doctorate and masters in Computer Science and Engineering from M.D. University, Rohtak in 2018 and 2010 respectively. Her topic of research is Software Reliability of Embedded System. Her areas of Interest are Software Engineering, Computer Networks, Operating System, Database Management Systems, and Artificial Intelligence etc. She has guided several research theses of UG and PG students in Computer Science and Engineering.

Vikram Bali (PhD) is Professor and Head-Computer Science and Engineering Department at JSS Academy of Technical Education, Noida, India. He had graduated from REC, Kurukshetra - B. Tech (CSE), Post-Graduation from NITTTR, Chandigarh - M.E (CSE) and doctorate (Ph.D) from Banasthali Vidyapith, Rajasthan. He has more than 20 years of rich academic experience. He has published more than 50 research papers in International Journals/Conferences and edited Books. He has authored Five textbooks. He has published Patent on Smart Dustbin- Sanitation \& Solid-Liquid Waste Management. He is on editorial and on the review panel of many International Journals. He is lifetime member of IEEE, Indian Society for Technical Education (ISTE), Computer Society of India (CSI) and Institution of Engineers (IE). He was Awarded Green Thinker Z-Distinguished Educator Award 2018 for remarkable contribution in the field of Computer Science and Engineering at 3rd International Convention on Interdisciplinary Research for Sustainable Development (IRSD) at Confederation of Indian Industry (CII), Chandigarh. He has also attended Faculty Enablement programme organised by Infosys and NASSCOM. He has been the member of board of studies of different Indian Universities and member of organizing committee for various National and International Seminars/Conferences. He is working on three sponsored research projects funded by TEQIP-3 and Unnat Bharat Abhiyaan. He has written books on Fundamental of "Cyber Security and Laws", "Software Engineering" and "Operating System". He is reviewer to many International Journals of repute like Inderscience and IGI Global. His research interest includes Software Engineering, Cyber Security, Automata Theory, CBSS and ERP.

Sudhir Mahajan $(P h D)$ is working as Assistant Professor in Department of ECE at PIET, Panipat. He has a vast teaching experience of 21 years for teaching engineering students. He has pursued his B. Tech. from SJCE, Mysore in Electronics and Communications Engineering (1993), M.Tech. from CGU University, Chhatisgarh (2005) and currently pursuing Doctorate from Mewar University. His area of research includes nano technology, wireless communication, microwave engineering, artificial intelligence etc. He has guided various innovative projects including smart cards, Nano electronics-based projects. 